\title{
Metastatic Gastric Cancer to the Colon
}

\author{
Eric Omar Then ${ }^{\mathrm{a}, \mathrm{b}, \mathrm{d}}$, Tyler Grantham ${ }^{\mathrm{c}}$, Xheni Deda ${ }^{\mathrm{b}}$, \\ Rajarajeshwari Ramachandran ${ }^{\mathrm{a}}$, Vinaya Gaduputi ${ }^{\mathrm{b}}$
}

\begin{abstract}
Gastric cancer is one of the most common and deadly cancers worldwide, especially amongst older males. Current data suggest gastric cancer is the fifth most common neoplasm and the third most deadly cancer, with an estimated 783,000 deaths in 2018. Risk factors associated with the development of gastric cancer include obesity, gastroesophageal reflux disease, Helicobacter pylori infection, and low socioeconomic status. Diagnosis of gastric cancer can be accomplished by endoscopy, which allows the clinician to obtain a biopsy specimen. Endoscopic ultrasound is also an important modality that is helpful in assessing tumor invasion. The most common sites of metastatic gastric cancer in descending order are the liver, peritoneum, lung and bone. Rarely will gastric cancer metastasize to the colon. Here we present a rare case of colonic metastasis of a primary gastric adenocarcinoma.
\end{abstract}

Keywords: Gastric cancer; Colon; Metastasis

\section{Introduction}

Gastric cancer is one of the most common and deadly cancers worldwide. Current data suggest gastric cancer is the fifth most common neoplasm and the third most deadly cancer worldwide, with an estimated 783,000 deaths in 2018 [1]. In the USA, the incidence of gastric cancer has been steadily declining in large part due to early treatment of Helicobacter pylori (H. pylori) infection. Although the annual US death rate decreased as well, the 5-year survival rate remains significantly elevated with reports of above $30 \%[2,3]$. The poor

Manuscript submitted March 28, 2021, accepted April 29, 2021

Published online July 10, 2021

aDivision of Gastroenterology and Hepatology, The Brooklyn Hospital Center, Clinical Affiliate of The Mount Sinai Hospital, Brooklyn, NY, USA

bDivision of Gastroenterology and Hepatology, SBH Health System, Bronx, NY, USA

'Department of Internal Medicine, St. George's University School of Medicine, Grenada, West Indies

${ }^{\mathrm{d} C o r r e s p o n d i n g ~ A u t h o r: ~ E r i c ~ O m a r ~ T h e n, ~ D i v i s i o n ~ o f ~ G a s t r o e n t e r o l o g y ~ a n d ~}$ Hepatology, The Brooklyn Hospital Center, Clinical Affiliate of The Mount Sinai Hospital, Brooklyn, NY, USA. Email: ericomarthen27@yahoo.com

doi: https://doi.org/10.14740/wjon1375 prognosis is associated with late stage of diagnosis as there is no standard or routine screening test for gastric cancer. Gastric cancer is metastatic at the time of diagnosis in 33\% of cases; however, metastasis to the colon is very rare [4]. This case study will report a deadly case of gastric cancer recurrence status post partial gastrectomy with colonic metastasis.

\section{Case Report}

The patient is a 66-year-old male with a medical history of hypertension and gastric cancer (status post partial gastrectomy 2 years prior) who presented to the emergency department (ED) complaining of progressively worsening peri-umbilical abdominal pain for 3 weeks and a 20-pound weight loss during that time. Regarding his past social history, the patient was married, lived with his family and was a former tobacco smoker (20 pack years). He denied alcohol or illicit drug use and had no known allergies. His physical exam showed a frail, cachectic male with a firm, distended, mildly tender abdomen with decreased bowel sounds. Additionally, there was a palpable mass in his left lower quadrant. He had clear breath sounds bilaterally with no rubs, murmurs or gallops auscultated on cardiac exam.

Notable laboratory workup included a complete blood count showed a white blood cell count of $3.8 \times 10^{3} / \mu \mathrm{L}$, hemoglobin of $8.7 \mathrm{~g} / \mathrm{dL}$, and a platelet count of $312 \times 10^{3} / \mu \mathrm{L}$. A comprehensive metabolic panel showed a normal creatinine of $1.1 \mathrm{mg} / \mathrm{dL}$, blood urea nitrogen of $21 \mathrm{mg} / \mathrm{dL}$ and electrolytes within normal limits. Given the patients symptoms a computed tomography (CT) scan of the abdomen with intravenous contrast was done and showed an apple core mass of the midtransverse colon measuring approximately $30 \times 44 \mathrm{~mm}$. There was also marked dilation of the colon proximal to this, consistent with large bowel obstruction (Fig. 1).

After obtaining the CT scan, the patient was admitted to the hospital ward for further management. The general surgery and gastroenterology services were consulted for recommendations. Initially an endoscopic placement of a palliative colonic stent was attempted but given the size of the lesion this was not amenable. General surgery then took the patient to the operating room and performed an en bloc resection of the colonic mass which was adherent to the transverse colon. The resected specimen was then sent to our pathologist for further evaluation. The results of the specimen showed metastatic adenocarcinoma consistent with gastric origin. This diagnosis was supported by cell positivity to cytokeratin $(\mathrm{CK}) 7$ and negativ- 


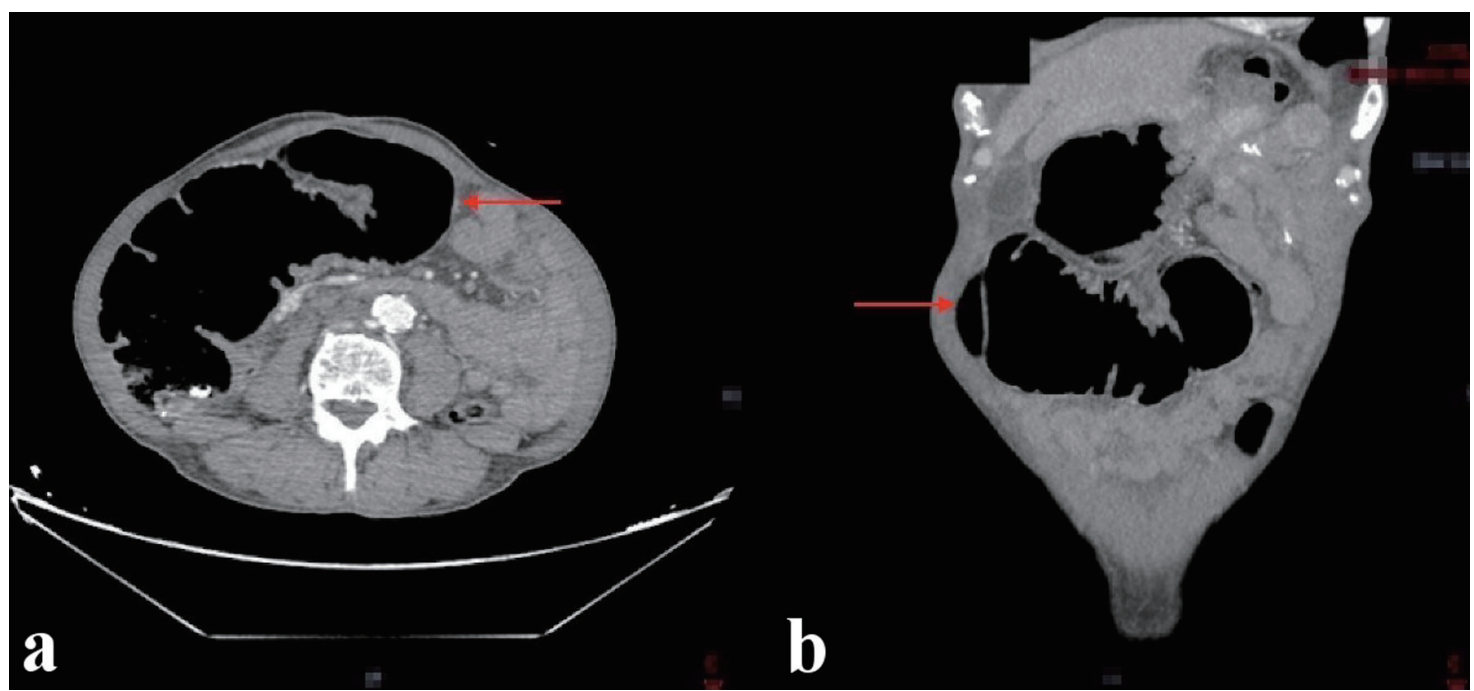

Figure 1. CT abdomen showing a mid-transverse colon mass measuring $30 \times 44 \mathrm{~mm}$ resulting in proximal colonic obstruction (red arrows). (a) Axial view. (b) Coronal view. CT: computed tomography.

ity to CK20 and CDX2 (Figs. 2, 3).

Postoperatively the patient's course was complicated by septic shock secondary to peritonitis and candidemia. Addi-
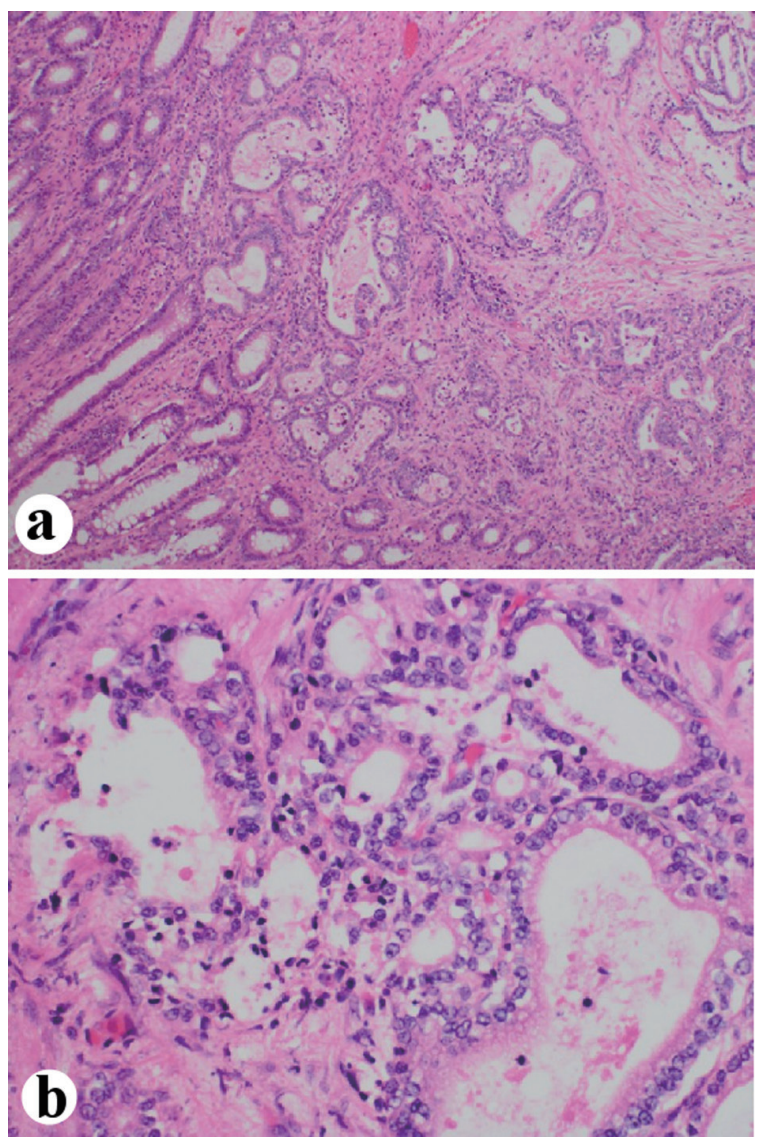

Figure 2. Colon mass biopsy specimen showing tumor cells infiltrating the muscularis propria and submucosa. (a) $\times 10$ magnification view. (b) $\times 40$ magnification view. tionally, the patient developed herpes zoster over his upper thorax. The infectious disease service was consulted, and the patient was started on broad-spectrum antibiotics. A few days the patient had not improved prompting a palliative care consult. Given his complicated course the patient's family opted to withdraw care and transfer the patient to a hospice facility for palliative care.

\section{Discussion}

A variety of risk factors are associated with the development of gastric cancer. H. pylori is thought to be the primary inciting factor for gastric cancer causing chronic inflammation in a stepwise pattern of disease progression. Over many years this leads to chronic gastritis, atrophic gastritis, intestinal metaplasia, dysplasia, and metaplasia eventually leading to cancer [5].

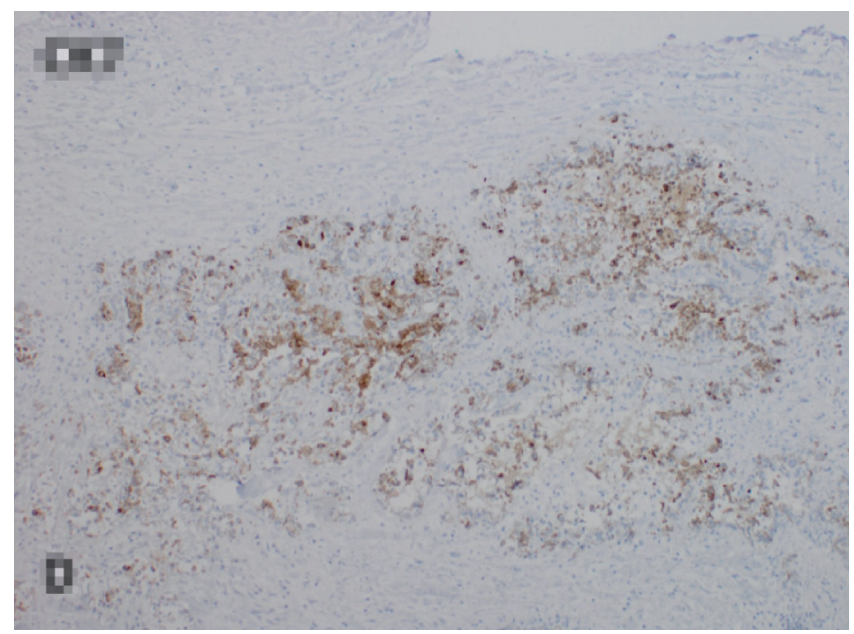

Figure 3. Tumor cells staining positively to CK7 and negative for CK20 and CDX2 (not shown), consistent with gastric origin. CK: cytokeratin. 
Epstein-Barr virus (EBV) is another pathogen that has been shown to contribute to gastric cancer in $10 \%$ of cases; however, the mechanism is not completely understood [6]. Familial causes comprise $10 \%$ of cases of gastric cancer. There are many different genetic mutations and molecular signaling pathways associated with gastric cancer; however, germline mutations in the E-cadherin ( $\mathrm{CDH1}$ ) gene are of particular interest and appear in 3\% of that population [6]. Environmental risk factors include obesity, cigarette smoking, alcohol use, and diets with high salt intake, red meat, and processed meats [6].

Esophagogastroduodenoscopy (EGD) with mucosal biopsy is the procedure of choice for diagnosis of gastric cancer. Signs and symptoms that warrant endoscopic evaluation include weight loss, abdominal pain, nausea and vomiting, early satiety, peptic ulcer symptoms, enlarged stomach, enlarged liver, or enlarged lymph nodes. Once identified, the mass or any abnormal mucosa suspicious for malignancy is targeted for biopsy with multiple specimens collected at the tissue site so that sampling incorporates deeper penetration into gastric tissue $[7,8]$. Double contrast barium swallow is another diagnostic consideration that can be done prior to EGD as it is non-invasive and cost-effective with high specificity for ruling out gastric cancer. If there are any malignant or unequivocal features or there are persistent symptoms after normal study, further diagnostic evaluation is necessary [8].

Endoscopic ultrasound (EUS) is another diagnostic tool can be used in conjunction with EGD, and is valuable in locoregional tumor, nodes, and metastases (TNM) staging. It provides information about depth of tumor invasion and the extent of perigastric lymphadenopathy. Its accuracy in T-staging varies between $60 \%$ and $90 \%$, and the N-staging accuracy ranges between $50 \%$ and $80 \%$ [9]. However, its application is limited in assessing the degree of metastasis and further staging requires cross-sectional imaging studies. CT scan is the imaging of choice.

The proportion of gastric cancer patients that present with metastasis has increased to over $40 \%$ in the last two decades [10]. Gastric cancer can metastasize by local extension, lymphatic spread, hematogenous spread, or peritoneal seeding. The most common sites of metastatic gastric cancer are the liver, peritoneum, and lymph nodes. Notable lymph nodes include (Sister Joseph's nodule) and left supraclavicular sentinel nodes (Virchow's node). Other common sites of metastasis are the ovaries (Krukenberg's tumor), lung, and bone [11]. Rarely will gastric cancer metastasize to the colon.

Patterns of metastasis have been shown to vary depending on the histologic type of gastric cancer. The Lauren classification describes two types of gastric adenocarcinoma: intestinal and diffuse. It has been shown that the intestinal type of gastric cancer is more likely to metastasize to the liver via the portal vein, while the diffuse type is more likely to spread to the peritoneum as well as the lymph nodes [12]. With rare metastasis to the colon, gastric cancer has been associated with Lauren's diffuse type histology, linitis plastica and peritoneal dissemination [13-18].

Recurrence is the most common reason for cancer-related death. Even when curative resection is possible, recurrence can be seen in approximately $60 \%$ of patients [4]. In Western countries, patients that undergo primary tumor resection and postoperative chemoradiation therapy have a 5-year survival of $40 \%$ [19]. Once there is evidence of recurrence, the patient is rarely curable. One Chinese study looked at gastric cancer patients who underwent a curative resection, to identify the factors associated with the recurrence patterns. Of the patients observed with recurrence, locoregional recurrence was shown in $85 \%$ of cases, peritoneal dissemination in $78 \%$ of cases, and distant metastasis in only $17 \%$ of cases. Distant metastasis was shown to be associated with the diffuse pattern of Lauren classification [4].

When recurrence with colonic metastasis is suspected, CT or alternative imaging techniques is recommended. A prompt colonoscopy should occur for possible intervention and subsequent diagnosis of metastasis via biopsy. With secondary metastatic lesions to colon, colonoscopy mainly shows lesion of the bowel wall, but can also show abnormal lumen appearance, such as an intraluminal obstructing lesion or multiple polypoid lesions. Other findings may be non-specific [20]. However, colonoscopy specific to gastric metastasis to the colon has been shown to be limited. A retrospective analysis of intestinal metastasis from gastric adenocarcinoma showed that in 13 patients that underwent colonoscopy with biopsies, positive results were obtained in only six patients. Those that were negative were proven to have metastasis by subsequent right hemicolectomy [13]. This may be in part due to the fact that metastatic carcinoma often preserves the mucosa layer so that further surgical biopsy is needed.

Surgery is the treatment of choice in gastric cancer limited to the stomach. The type of surgery depends on the location of the tumor and depth of invasion, and includes endoscopic mucosal resection, distal esophagectomy, and subtotal or total gastrectomy. In metastatic disease however, treatment with chemotherapy and targeted radiotherapy should be pursued. The first line chemotherapy consists of platinum-based agents and a cytotoxic compound such as 5-fluorouracil (5FU) with or without trastuzumab (if human epidermal growth factor receptor-2 (HER2) is overexpressed) [6]. A multitude of molecular signaling pathways have shown to be involved in the pathogenesis of gastric cancers and thus further targeted therapy may be warranted. These include tyrosine kinase inhibitors, cell structure remodeling therapies, targeting of deoxyribonucleic acid (DNA) damage repair proteins and immunotherapy. However, many of these current treatment strategies have poor patient performance status and are accompanied by high grade toxicity [21]. In these clinical scenarios where the risks outweigh the benefits, such as in our case, palliative care and best supportive care are the appropriate treatment options.

\section{Conclusions}

Gastric cancer remains one of the deadliest cancers worldwide. Metastasis and recurrence are very common, but rarely will present with metastasis to the colon. Colonic metastasis is commonly associated with the diffuse type of adenocarcinoma, whereas the intestinal type has been shown to metastasize elsewhere. Endoscopy coupled EUS are imperative in diagnosing and subsequent staging to dictate further manage- 
ment. CT scan and colonoscopy are important in visualizing intestinal metastasis, but colonoscopy with biopsy may have limitations to diagnosis and may warrant surgical biopsy. Once recurrence is confirmed the prognosis is grim. Although multiple chemotherapeutic and alternative target-based therapies exist, one must consider the patients best interest in determining the plan of care.

\section{Acknowledgments}

None to declare.

\section{Financial Disclosure}

No funding to disclose.

\section{Conflict of Interest}

The case report was presented in the form of an abstract (poster) in the American College of Gastroenterology Conference in San Antonio, TX (October 2019), USA.

\section{Informed Consent}

Written informed consent was obtained by the patient's next of kin (patient expired).

\section{Author Contributions}

EOT, XD, and RR involved in conception and design. EOT, TG and XD drafted the article. VG critically revised the article for important intellectual content. EOT, TG, XD, RR, and VG involved in the final approval of the article. All Authors have made contributions to the article and have reviewed it before submission.

\section{Data Availability}

The authors declare that data supporting the findings of this study is available within the article.

\section{References}

1. Bray F, Ferlay J, Soerjomataram I, Siegel RL, Torre LA, Jemal A. Global cancer statistics 2018: GLOBOCAN estimates of incidence and mortality worldwide for 36 cancers in 185 countries. CA Cancer J Clin. 2018;68(6):394424.

2. Milano AF. 20-Year comparative survival and mortality of cancer of the stomach by age, sex, race, stage, grade, cohort entry time-period, disease duration \& se- lected ICD-O-3 oncologic phenotypes: a systematic review of 157,258 cases for diagnosis years 1973-2014: (SEER*Stat 8.3.4). J Insur Med. 2019;48(1):5-23.

3. Siegel RL, Miller KD, Jemal A. Cancer statistics, 2020. CA Cancer J Clin. 2020;70(1):7-30.

4. Deng JY, Liang H. Clinical significance of lymph node metastasis in gastric cancer. World J Gastroenterol. 2014;20(14):3967-3975.

5. Correa P, Haenszel W, Cuello C, Zavala D, Fontham E, Zarama G, Tannenbaum S, et al. Gastric precancerous process in a high risk population: cohort follow-up. Cancer Res. 1990;50(15):4737-4740.

6. Sexton RE, Al Hallak MN, Diab M, Azmi AS. Gastric cancer: a comprehensive review of current and future treatment strategies. Cancer Metastasis Rev. 2020;39(4):11791203.

7. ASGE Standards of Practice Committee, Evans JA, Chandrasekhara V, Chathadi KV, Decker GA, Early DS, Fisher $\mathrm{DA}$, et al. The role of endoscopy in the management of premalignant and malignant conditions of the stomach. Gastrointest Endosc. 2015;82(1):1-8.

8. Layke JC, Lopez PP. Gastric cancer: diagnosis and treatment options. Am Fam Physician. 2004;69(5):1133-1140.

9. Papanikolaou IS, Triantafyllou M, Triantafyllou K, Rosch T. EUS in the management of gastric cancer. Ann Gastroenterol. 2011;24(1):9-15.

10. Riihimaki M, Hemminki A, Sundquist K, Sundquist J, Hemminki K. Metastatic spread in patients with gastric cancer. Oncotarget. 2016;7(32):52307-52316.

11. Hess KR, Varadhachary GR, Taylor SH, Wei W, Raber MN, Lenzi R, Abbruzzese JL. Metastatic patterns in adenocarcinoma. Cancer. 2006;106(7):1624-1633.

12. Esaki Y, Hirayama R, Hirokawa K. A comparison of patterns of metastasis in gastric cancer by histologic type and age. Cancer. 1990;65(9):2086-2090.

13. Jang HJ, Lim HK, Kim HS, Cho EY, Lee SJ, Kim KA, Choi D. Intestinal metastases from gastric adenocarcinoma: helical CT findings. J Comput Assist Tomogr. 2001;25(1):61-67.

14. Pace U, Contino G, Chiappa A, Bertani E, Bianchi PP, Fazio N, Renne G, et al. Metachronous colon metastases from gastric adenocarcinoma: A Case Report. Case Rep Oncol. 2009;2(2):92-96.

15. Fisher ER, Brown CH. Linitis plastica carcinoma of the stomach with extensive metastases simulating a colonic lesion. Gastroenterology. 1952;20(3):503-508.

16. Lee HC, Yang MT, Lin KY, Tu HY, Zhang TA, Chen PH. Metastases from gastric carcinoma to colon in the form of multiple flat elevated lesions: a case report. Kaohsiung J Med Sci. 2004;20(11):552-557.

17. Elghali MA, Fadhl H, Hamila F, Mestiri S, Jarrar MS, Letaief R. Colon Metastasis, 8 years after Gastrectomy, for Stage I Gastric Cancer. J Gastrointest Cancer. 2019;50(1):127-129.

18. Dixon CF, Stevens GA. Carcinoma of Linitis Plastica type involving the intestine. Ann Surg. 1936;103(2):263272.

19. Rohatgi PR, Yao JC, Hess K, Schnirer I, Rashid A, Mansfield PF, Pisters PW, et al. Outcome of gastric cancer 
patients after successful gastrectomy: influence of the type of recurrence and histology on survival. Cancer. 2006;107(11):2576-2580.

20. Galanopoulos M, Gkeros F, Liatsos C, Pontas C, Papaefthymiou A, Viazis N, Mantzaris GJ, et al. Secondary metastatic lesions to colon and rectum. Ann Gastroenterol. 2018;31(3):282-287.

21. Lazar DC, Taban S, Cornianu M, Faur A, Goldis A. New advances in targeted gastric cancer treatment. World J Gastroenterol. 2016;22(30):6776-6799. 